\title{
Membranes with a microparticulate morphology
}

\author{
Tai-Horng Young* \\ Center for Biomedical Engineering. College of Medicine, National Taiwan University, \\ Taipei 10016, Taiwan, Republic of China
}

and Leo-Wang Chen

Department of Chemical Engineering, National Taiwan University, Taipei 10764, Taiwan, Republic of China

and Liao-Ping Cheng

Department of Chemical Engineering, Tamkang University, Taipei, Taiwan, Republic of China

(Received 17 May 1995)

\begin{abstract}
Ethylene-vinyl alcohol (EVAL) membranes with particles-bonded structure are prepared by phase inversion from DMSO solvent into the coagulation baths of water and isopropanol (IPA) mixture. It is based on controlling the relative diffusion rate of solvent to nonsolvent and the liquid-liquid demixing area. Using this analysis, it can explain the effects of adding IPA to the water bath on the formation of particles and the suppression of macrovoids. The results proposed here are believed to be valid also for other systems. Copyright $(\mathbb{C} 1996$ Elsevier Science Ltd.
\end{abstract}

(Keywords: particles-bonded; diffusion; liquid-liquid demixing)

\section{INTRODUCTION}

Since the development of asymmetric type membrane by Loeb and Sourirajan ${ }^{1}$, the field of membrane science and technology has been experiencing an increasing growth in separation science. The performance of the membrane is principally governed by the characteristics of the polymer itself and the preparation of the membrane. Nowadays, membranes are generally prepared by phase inversion method ${ }^{2}$. In this process, a casting solution consisting of polymer and solvent is immersed into a nonsolvent coagulation bath. Exchange of solvent and nonsolvent due to diffusion causes the casting solution to go through a phase transition, by which the membrane is formed. Improvements have made possible a great variety of biological applications for membrane systems.

In 1979 Yamashita et al. utilized ethylene-vinyl alcohol (EVAL) copolymer to produce membranes having improved permeability characteristics for the dialysis of blood ${ }^{3}$. The structure of their membranes characterized by constituent particles bonded to each other with an average diameter within the range of from 100 to $1000 \AA$. Then Sakurada et al. ${ }^{4}$ reported that hemodialyzer using EVAL membrane can conduct nonanticoagulant hemodialysis and exhibits improved efficacy for renal failure patients. Although many factors affect the blood compatibility and biocompatibility of membranes, we consider the particles-bonded structure may play an important role. There are few references about

\footnotetext{
* To whom correspondence should be addressed
}

the formation of particles mentioned in literature: Cheng ${ }^{5}$ suggests that the simultaneous liquid-liquid phase separation and crystallization result in the particles morphology. However, some crystalline polymer does not have the particles-bonded structure. Boom et $a l .{ }^{6}$ report that the appearance of a nodular structure follows a mechanism of spinodal decomposition. However, it only can explain the nodular structure appearing in the top layer of ultrafiltration membrane during the very early stage of the immersion step. Therefore, the purpose of this paper is to describe the formation mechanism of particles-bonded membranes and to provide better control of membrane formation techniques.

\section{THEORY}

Before investigating the mechanism of particles-bonded membranes, we will first summarize here the important points and some results of membrane formation.

Clearly the membrane forming process of the phase inversion process is controlled by thermodynamic properties and diffusion kinetics of the system. The thermodynamics should explain the effects of different ternary system on the construction of a phase diagram. An isothermal ternary phase diagram is presented in Figure 1. The main route of phase inversion process involves two different types of phase transition ${ }^{7}$ : (i) liquid-liquid phase separation (from I to II) - the completely miscible solution crosses the binodal boundary to enter the twophase region; (ii) solidification (from I to III or from II to III) - an assumed boundary is located in the diagram 

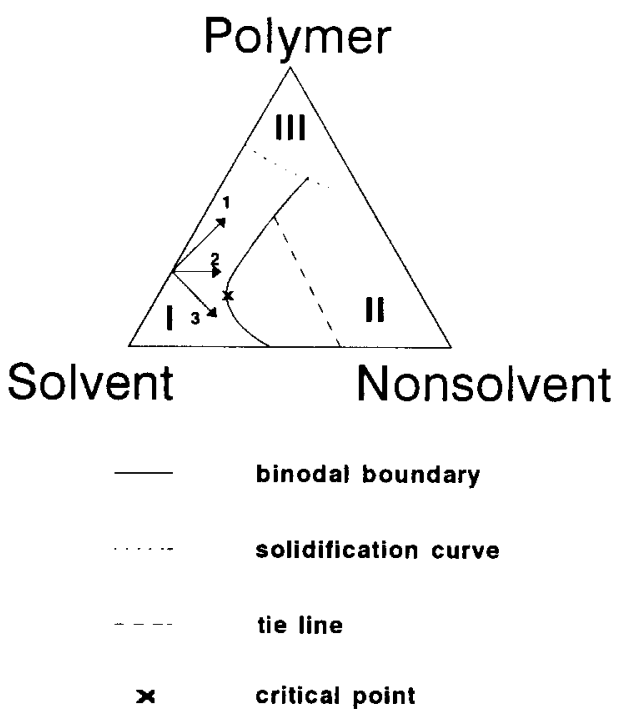

Figure 1 Ternary phase diagram of EVAL-DMSO-water system. I: homogeneous solution region; II: two-phase region; III: solidification region

since the viscosity of the polymer solution increases to a certain assumed value, the motion of polymer chains will be limited and the system can be regarded as a solid to fix the membrane structure.

Previously, Altena and Smolders ${ }^{8}$, and Yilmaz and $\mathrm{McHugh}^{9}$ have calculated binodals and tie lines for some ternary systems using the Flory-Huggins theory. Boom et al. ${ }^{10}$ proposed a linearized cloudpoint correlation that is only caused by liquid-liquid demixing. From the relation, they suggest that the situation of the critical point depends on the molecular weight of polymer, and the liquid-liquid demixing gap becomes smaller when the nonsolvent is weaker.

The phase diagram is the description of an equilibrium state. In polymer systems, however, the phase separation is largely governed by kinetic parameters. The mass transfer property mainly involves the relative diffusion rates of solvent and nonsolvent in the polymer solution. Some investigators have carried out different models to describe the variation of concentration of the immersed film ${ }^{11-15}$. The models regard the immersed film as a whole to calculate the composition paths in the phase diagram. However, this profile is only valid for the first instances after immersion and the distance close to the film-bath interface. In our opinion ${ }^{16}$, it is convenient and appropriate to discuss the composition paths in the phase diagram by dividing the membrane into $N$ layers. By assuming the diffusion ratio of nonsolvent to solvent a constant in each layer, the composition path of each layer will be a straight line in the ternary phase diagram. Therefore, the membrane structure is determined by the diffusion ratio of nonsolvent to solvent of each layer. If nonsolvent diffuses into the casting solution more rapidly than solvent diffuses out in some layer, the composition path of the layer may cross the binodal to cause liquidliquid phase separation (line 2 in Figure 1) and the result is a porous layer. On the other hand, if the composition path of the layer enters the solidification (line 1 in Figure 1) region directly, then a dense structure is formed.

It has been suggested that the membrane formation process can simplify the $N$-layer model as a two-step mechanism ${ }^{16-18}$. The membrane consists of two layers: top layer and sublayer. When the casting solution is immersed into the coagulation bath, the top layer is formed first at the casting solution-nonsolvent interface. The ratio of nonsolvent inflow to solvent outflow determines the local composition of the top layer structure at the instant of phase transition. The structure of the sublayer also depends on the local composition at the instant of phase transition. However, it is directly influenced by the degree of aggregation of the polymer molecules at the top layer. If the top layer structure is relatively compact, it will increase the barrier for diffusion of solvent from and nonsolvent into the sublayer of casting solution. Therefore, the dense top layer determines the diffusion rate of both solvent and nonsolvent molecules through the film and thus the composition path of the sublayer. On the other hand, if the top layer structure is microporous, mass transfer rate is only slightly influenced by the top layer. As a consequence, the phase transition in the sublayer is the same as that in the top layer and the two-step mechanism is reduced to one-step mechanism. So the membrane structure can be considered as homogeneous.

\section{The mechanism of pore formation and particle formation}

A typical asymmetric membrane shows a dense skin with a porous sublayer. From the above two-step mechanism model, a dense skin is formed because solvent in the casting solution desolvates rapidly into the coagulation bath and relatively little nonsolvent diffuses into the casting solution at the moment the casting solution and nonsolvent come into contact. Consequently, the top layer directly enters the solidification region and polymer molecules at the interface aggregate rapidly to form a dense skin where no pore exists.

The thickness of the skin layer will increase gradually until the diffusion of solvent from the sublayer solution through the dense skin layer into the coagulation bath is restrained. As a result, the composition of the sublayer does not change as rapidly and the solution is within the initial homogeneous solution region. In the meantime, nonsolvent diffuses through the dense top layer. When a certain amount of nonsolvent has caused the composition variation to cross the binodal boundary to become unstable somewhere in the sublayer, the nucleus of polymer-poor phase occurs to form the nascent pore. Depending on the polymer concentration of the phase transition, the polymer-rich phase or polymer-poor phase separates from the casting solution. If the polymer concentration of the phase transition is smaller than the critical point, the polymer-rich phase separates as a nucleus of a particle rather than a void. In general, the location of the critical point is at low polymer concentration. Hence the binodal and the composition path reach above the critical point, indicating that a tendency to nucleation of the polymer-poor phase.

A nascent pore will grow if nonsolvent can continually diffuse into it to induce solvent surrounding the nascent pore to diffuse into it. The diffusion of solvent into the growing pore will cause an increase in the polymer concentration of the polymer-rich phase surrounding the pore. When the surrounding polymer-rich phase enters the solidification region, a pore stops their growth, i.e. the solidification is the major factor determining pore wall structure. 


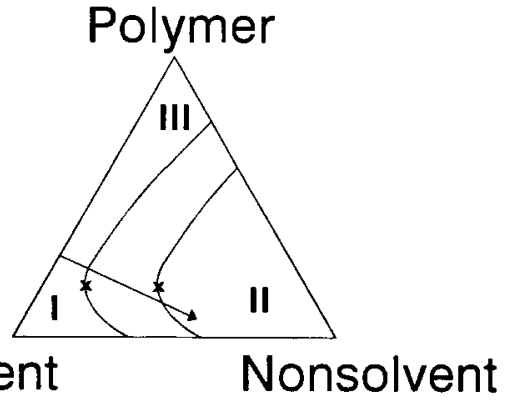

Figure 2 Ternary phase diagram of particles-bonded membrane (demixing area becomes smaller)

Clearly the polymer-poor phase contains solvent and nonsolvent. Thus, in a system with a large driving force for nonsolvent and solvent, only a little nonsolvent into a pore is sufficient to introduce more solvent being depleted from the polymer solution surrounding the pore to maintain the equilibrium request of the polymer-poor phase. Since polymer molecules are clustered together to exclude solvent from within their domains, a small pore rapidly grows up to a macrovoid. Therefore, if many nuclei are initiated at the same time then the growth of every nucleus will be limited by other nearby nuclei because every nucleus consumes solvent. In this way, the growth of macrovoid is impossible and only smaller pores are formed. Smolders et al. ${ }^{19}$ also proposed a similar explanation for void formation. They suggest that macrovoids are formed if the solvent concentration in new nuclei exceeds a certain minimum value. We do agree with their suggestion. However, we believe that the solvent concentration in new nuclei is determined by the interaction between solvent and nonsolvent.

As has been mentioned above it would seem that particle structure occurs when the composition path moves towards the right lower side to cross the binodal below the critical point (line 3 in Figure 1). Therefore, there are two conditions that must be satisfied:

(1) The rate of diffusion of nonsolvent into the casting solution should be large relative to that of solvent into the coagulation bath.

(2) The critical point should not be in the neighborhood of the solvent-nonsolvent axis and the liquid-liquid demixing area should decrease (see Figure 2).

Then the composition path may cross the binodal boundary at much lower polymer concentration to induce nucleation of particles.

\section{EXPERIMENTAL}

\section{Materials}

EVAL copolymer that has a content of $56 \mathrm{~mol} \%$ of vinyl alcohol monomeric units was obtained using previously reported techniques ${ }^{20}$. Deionized and ultrafiltrated water was used in our experiments. Other reagents were of chemical reagent grade and were used without further purification.

\section{Membrane preparation}

The EVAL copolymers were dissolved in DMSO to form a $25 \%$ polymer solution. The solution was spread
Table 1 Summary of experimental data

\begin{tabular}{ll}
\hline Membrane & Nonsolvent composition \\
\hline 1 & Pure water \\
2 & $75 \%$ water $25 \%$ IPA \\
3 & $50 \%$ water $/ 50 \%$ IPA \\
4 & $25 \%$ water $/ 75 \%$ IPA \\
5 & Pure IPA \\
\hline
\end{tabular}

on glass plates in a uniform thickness of about $175 \mu \mathrm{m}$. The glass plates were immersed immediately into the coagulation bath of mixture of water and isopropanol (IPA). No evaporation step is performed before the casting solution immersed into the coagulation bath. After polymer precipitated, the membrane was removed from the glass plate and kept in a pure water bath. The morphology of the membranes was examined using a scanning electron microscope (SEM). Table $l$ is a summary of the experimental data.

\section{RESULTS}

The ternary phase diagram of EVAL-DMSO-water has been reported in the literature ${ }^{20}$. The critical point is not in the neighbourhood of the solvent-nonsolvent axis. According to the theory of Boom et al. ${ }^{10}$, in case of adding IPA to the water coagulation bath, the liquidliquid demixing gap will vary depending on the interaction between polymer and nonsolvent mixture. However, the same molecular weight of EVAL will still maintain the situation of the critical point in the same concentration of polymer in the ternary phase diagram.

When adding IPA to the water coagulation bath, the affinity between EVAL copolymer and nonsolvent mixture initially increases, then goes through a maximum about at $35-50 \%$ of IPA nonsolvent mixture, and finally declines ${ }^{17}$. Thus, as the IPA content of the coagulation bath increases, the demixing gap decreases initially and then increases. In the same way, the diffusion tendency of nonsolvent into the EVAL solution is influenced by the affinity between EVAL copolymer and nonsolvent mixture, so it increases initially and then decreases. On the contrary, the DMSO outflow decreases continually with increasing the IPA content in the coagulation bath due to IPA reducing the strong interaction between DMSO and water $^{17}$. Therefore the composition path will change from line 1 to 2 or even to 3 to reach the binodal at a lower polymer concentration, see Figure 1 . Liquid-liquid demixing starts and a two-phase system consisting of concentrated polymer droplets (nuclei) dispersed in a dilute polymer solution is possible. The nuclei formed will grow further until thermodynamic equilibrium is reached. In this way a membrane with particle-bondedtogether structure is obtained.

Membrane 1 using water as nonsolvent shows a dense skin with a finger-like structure (SEM pictures were presented in Figure 2 of ref. 17). Due to the strong interaction between DMSO and water, DMSO in the casting solution desolvates rapidly into the water coagulation bath at the moment the casting solution and come into contact. However, relatively little water diffuses into the casting solution since it is nonsolvent for EVAL copolymer. Consequently, EVAL molecules at the interface aggregate rapidly form a dense skin without pores. 
As shown in line 1 of Figure 1 , the casting solution enters the solidification region directly. The diffusion of DMSO into the water coagulation bath from the sublayer solution is much slower than from the top layer, because DMSO molecules must diffuse through

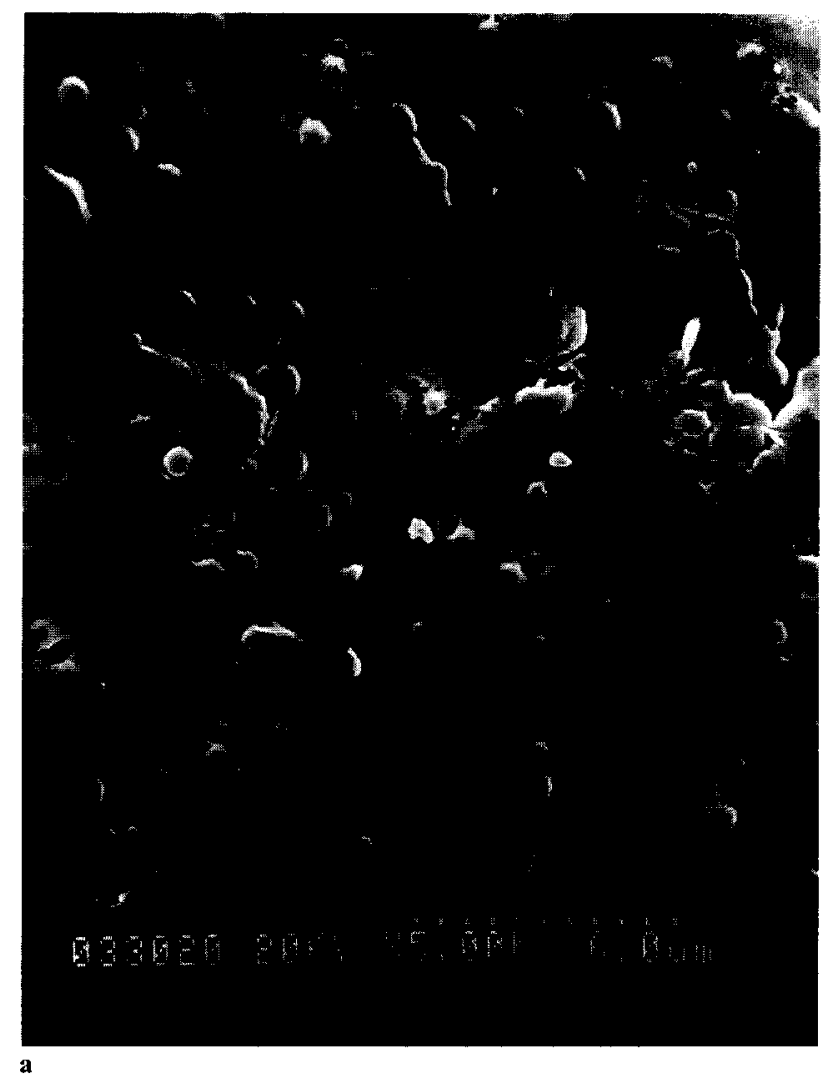

a
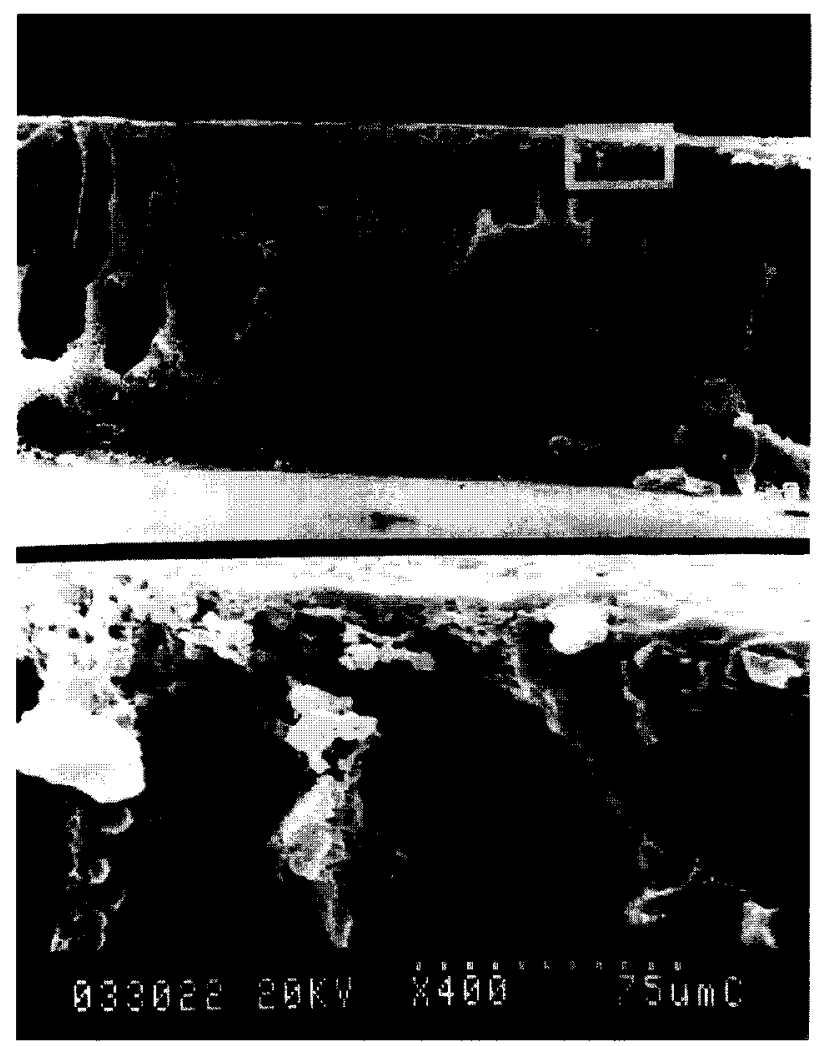

b

Figure 3 SEM pictures of membrane 2, (a) top and (b) cross-section the dense skin layer. As a result, water molecules have enough time to diffuse into the interior portion of the casting solution to initiate nuclei of polymer-poor phase. These pores may grow rapidly by much DMSO diffusing into pores with a large driving force for DMSO and
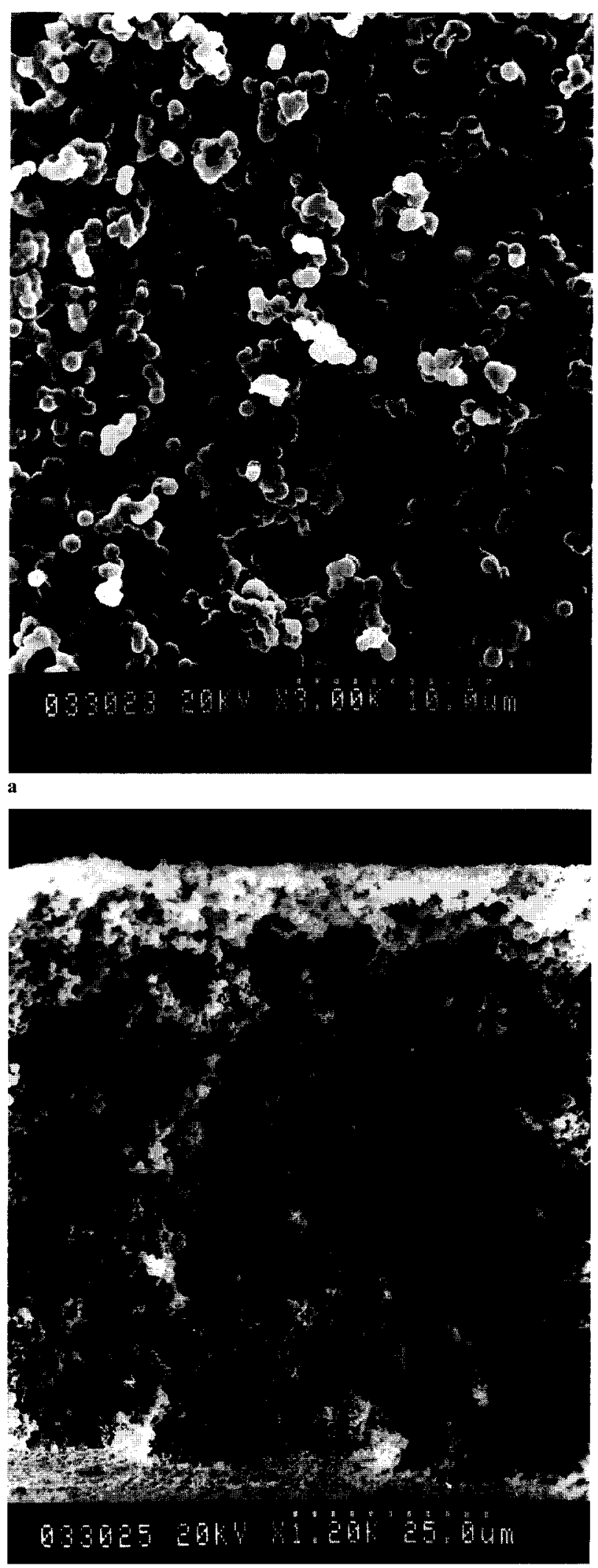

b

Figure 4 SEM pictures of membrane 3, (a) top and (b) cross-section 
water. Thus finger-like cavities are clearly observed in membrane 1.

Figure 3 shows that membrane 2, coagulated in a IPA-water bath containing $25 \%$ IPA, has some pores in the top layer. This is attributed to the fact that it has a weaker interaction between DMSO and nonsolvent mixture than membrane 1. DMSO outflow decreases and the nonsolvent has more time to diffuse into the surface solution. Therefore the slope of the composition path will become smaller and may enter the liquid-liquid phase separation region, such as line 2 in Figure 1.

Since the top layer is not very porous, it does not lead the large amount of nonsolvent into the sublayer to initiate many nuclei formations. Therefore, a freshly formed nucleus has potential to grow larger dimensions. In a manner similar to that observed for the top layer, DMSO and nonsolvent mixture do not have an interaction strong enough to introduce more DMSO being depleted from the polymer solution surrounding the already formed nucleus; thus the sublayer cannot form complete finger-like structures as do membrane 1, coagulated in a water bath.

When adding IPA to the water coagulation bath up to a composition of $50 \%$, the structure of membrane 3 shows a vast difference from that of membrane 1 and membrane 2. Not only does the particle structure occur in the top layer, but the overall structure of membrane forms the constituent particles about ranging from 0.5 to $1 \mu \mathrm{m}$ units, see Figure 4. Finger-like structure disappears completely. For membrane 3 the polymer and the nonsolvent system are the most compatible in the membranes $1-5$, so the liquid-liquid demixing gap becomes the smallest. In addition, the diffusion rate of the weakest nonsolvent mixture is the fastest one and the DMSO outflow decreases continually. Therefore the composition path will reach the binodal at a lower polymer concentration, even smaller than the critical solution concentration; see Figure 2. Consequently, concentrated polymer droplets disperse in a dilute polymer solution to form the particle structure. Of course the particle structure of the top layer forms first. The diffusion rate of DMSO from the underlying solution is about the same as from the surface solution, since the space between the neighboring particles is large enough not to greatly influence the diffusion rate. Nonsolvent also may enter the underlying solution by diffusion through the upper surface to induce the similar liquid-liquid phase separation. In this way the resulting membrane is with total particlesbonded structure.

When the IPA content of the coagulation bath is increased to $75 \%$, the particles are clearly reduced in membrane 4 (see Figure 5). Both pores and particles exist in the top layer. This phenomenon results from the fact that the demixing region increases and the diffusion tendency of nonsolvent into the casting solution decreases because EVAL and nonsolvent mixtures become more incompatible. So the phase transition of membrane 4 occurs in the higher polymer concentration than that of membrane 3 and is still near the critical point. Membrane 4 also occurs simultaneously with incomplete finger-like structures next to the top layer, similar to membrane 2 . However, for membrane 4 , there is a relatively weaker interaction between solvent and nonsolvent mixture than for membrane 2. Furthermore, the large amount of nonsolvent can diffuse through porous top layer into
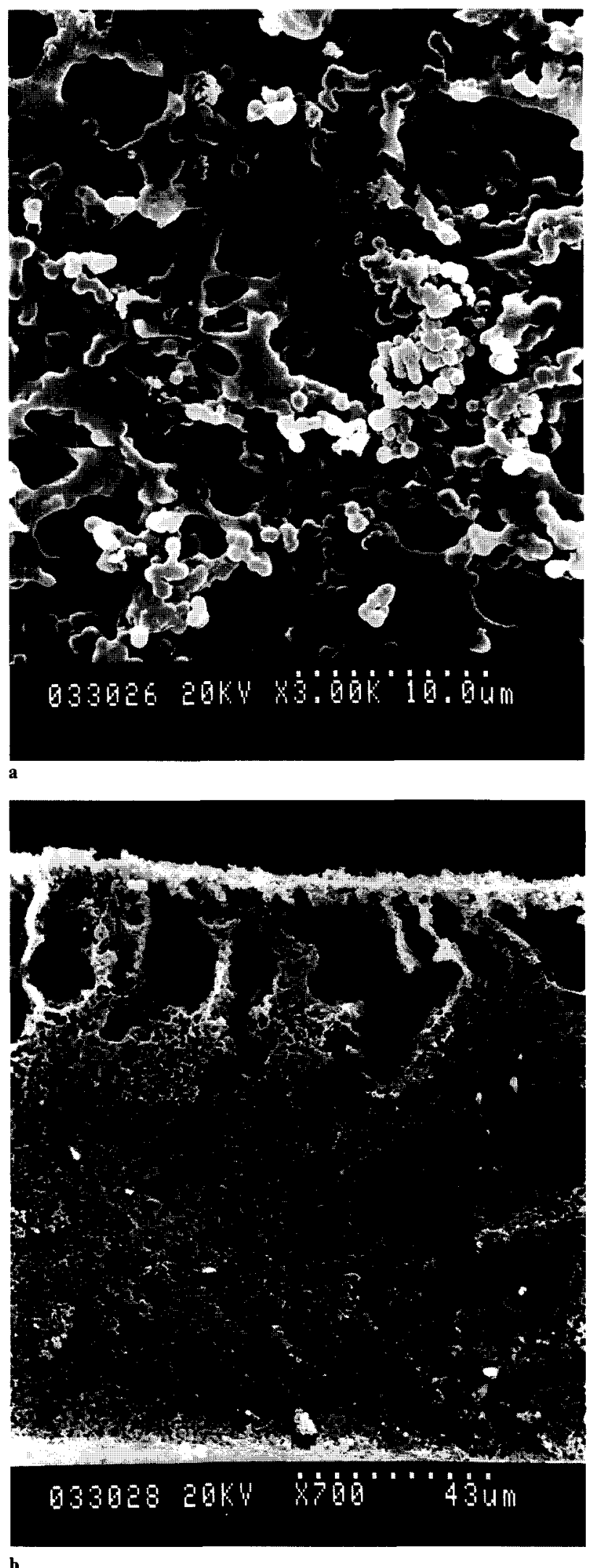

Figure 5 SEM pictures of membrane 4, (a) top and (b) cross-section

the sublayer to initiate many nuclei to limit their growth. Thus the fingers become smaller.

When the coagulation bath contains IPA only, the diffusion rate of DMSO into the coagulation bath is 


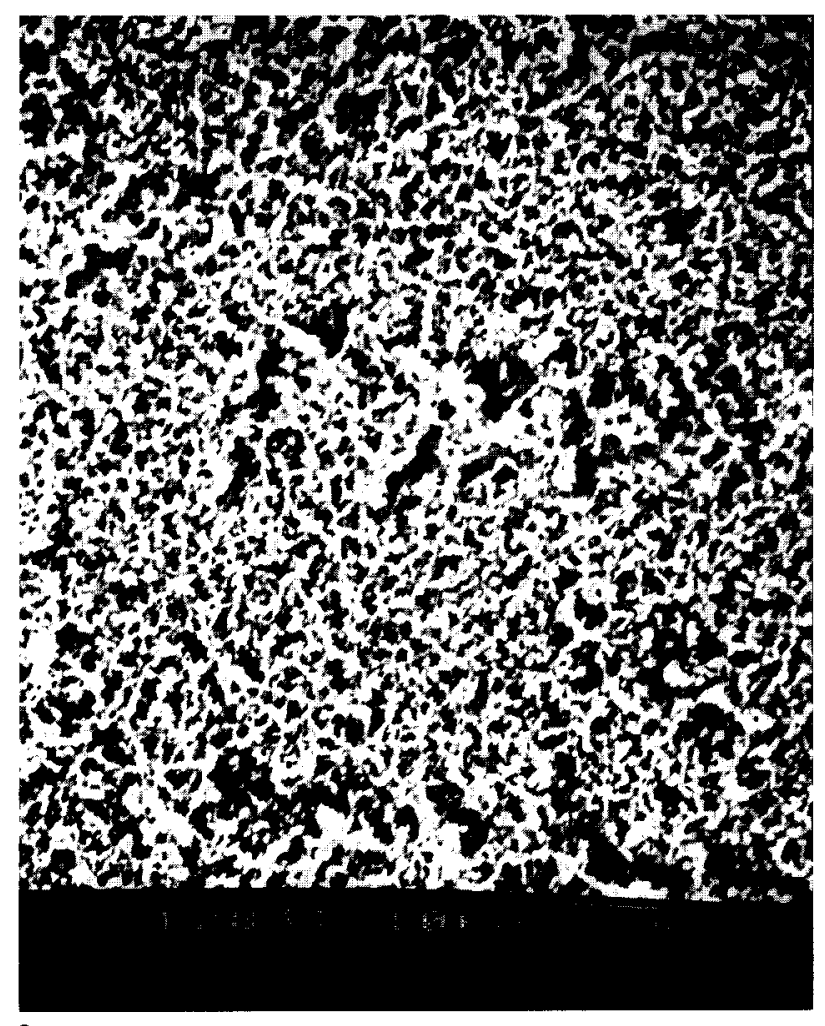

a

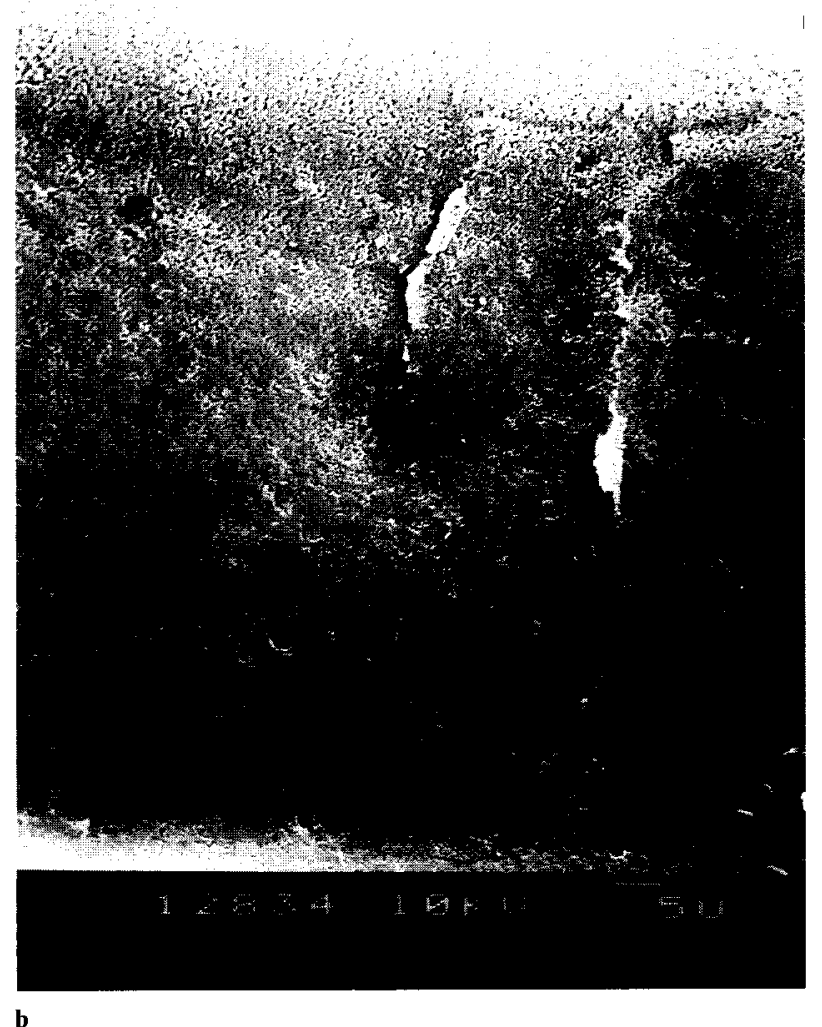

Figure 6 SEM pictures of membrane 5, (a) top and (b) cross-section

small owing to the weak interaction between DMSO and IPA. It results that the top layer of the casting solution maintains the dispersed state and does not precipitate rapidly. Hence IPA has enough time to diffuse into the surface solution. Since its demixing gap will be larger than that of using water-IPA mixture as nonsolvent, the composition path enters the binodal above the critical point and membrane 5 forms a porous surface; see Figure 6. As mentioned above, both DMSO and nonsolvent molecules can easily pass through the porous top layer, so the sublayer proceeds along the same transition to form the porous structure. Because no water is present in the coagulation bath to keep stronger interaction between solvent and nonsolvent mixture, the pores will not grow to form macrovoid.

\section{CONCLUSIONS}

It is clearly shown that by adding IPA to the water bath, membrane morphology can be changed from the typical asymmetrical structure to particles-bonded structure. It is based on controlling the relative diffusion rate of nonsolvent to solvent and the liquid-liquid demixing area. If we can increase the diffusion rate of nonsolvent relative to that of solvent and decrease the demixing area, we can let the composition path cross the binodal boundary below the critical point and obtain the particles-bonded structure. From these results, by controlling the preparation of membranes, membranes with different structures may be obtained for different applications.

\section{ACKNOWLEDGEMENT}

The authors thank the National Science Council of the Republic of China for their financial support by project NSC 84-2216-E-002-016.

\section{REFERENCES}

1 Locb, S. and Sourirajan. S. Adv. Chem. Ser. 1963, 38, 117

2 Kesting, R.E. 'Synthetic Polymeric Membranes', Wiley, New York, 1985

3 Yamashita, S., Nagata, S. and Takakura. K. U.S. Patent 4,134,837, 1979

4 Sakurada, Y., Sueoka, A. and Kawahashi, M. Polymer J. 1987, 19. 501

5 Cheng L. P., 'Biomedical Engineering: Applications, Basis, Communication', Vol. 5. p. 796, 1993

6 Boom, R. M., Wienk, I. M., van den Boomgaard, Th. and Smolders, C. A. J. Membrane Sci. 1992, 73, 277

7 Young, T.-H. and Chen, L.-W. J. Membrane Sci. 1991, 57, 69

8 Altena. F. W. and Smolders, C. A. Macromolecules 1982, 15, 1491

9 Yilmaz, L. and McHugh, A. J. J. Appl. Polym. Sci. 1986, 31, 997

10 Boom, R. M., van den Boomgaard. Th., van den Berg, J. W. A. and Smolders, C. A. Polymer 1993, 34, 2348

11 Cohen, C., Tanny, G. B. and Prager, S.J. J. Polvm. Sci., A-2 $1979,17,477$

12 Yilmaz, L. and McHugh, A. J. J. Membrane Sci. 1986, 28, 287

13 Reuvers, A. J., van der Berg, J. W. A. and Smolders, C. A. J. Membrane Sci. 1987, 34, 45

14 Tsay, C. S. and McHugh. A. J. J. Polym. Sci., Polvm. Phys. Edn 1990, 28, 1327

15 Radov, P., Thicl, S. W. and Hwang, S.-T. J. Membrane Sci. $1992,65,213$

16 Young, T.-H. and Chen, L.-W. J. Membrane Sci. 1991, 59, 169

17 Chen, L.-W. and Young, T.-H. J. Membrane Sci. 1991, 59, 15

18 Young, T.-H. and Chen, L.-W. J. Membrane Sci. 1993, 83, 153

19 Smolders, C. A., Reuvers, A. J., Boom, R. M. and Wienk, I. M. J. Membrane Sci. 1992, 73, 259

20 Chen. L.-W. and Young, T.-H. Makromol., Chem. Macromol. Symp. 1990, 33, 183 